\title{
Vitamin D status and longitudinal lung function decline in the Lung Health Study
}

\author{
K.M. Kunisaki*, D.E. Niewoehner*,\#, R.J. Singh` and J.E. Connett ${ }^{+}$
}

ABSTRACT: Low blood vitamin $D$ levels have been postulated to be a risk factor for worse lung function, based largely on cross-sectional data. We sought to use longitudinal data to test the hypothesis that baseline plasma 25-hydroxyvitamin D (25(OH)D) is lower in subjects with more rapid lung function decline, compared to those with slow lung function decline.

We conducted a nested, matched case-control study in the Lung Health Study 3 cohort. Cases and controls were continuous smokers with rapid and slow lung function decline, respectively, over $\sim 6$ yrs of follow-up. We compared baseline 25(OH)D levels between cases and controls, matching date of phlebotomy and clinical centre.

Among 196 subjects, despite rapid and slow decliners experiencing strikingly and significantly different rates of decline of forced expiratory volume in $1 \mathrm{~s}\left(-152\right.$ versus $\left.-0.3 \mathrm{~mL} \cdot \mathrm{yr}^{-1} ; \mathrm{p}<0.001\right)$, there was no significant difference in baseline $25(\mathrm{OH}) \mathrm{D}$ levels $\left(25.0\right.$ versus $\left.25.9 \mathrm{ng} \cdot \mathrm{mL}^{-1} ; \mathbf{p}=0.54\right)$. There was a high prevalence of vitamin D insufficiency (35\%) and deficiency (31\%); only $4 \%$ had a normal 25(OH)D level in the winter.

Although vitamin D insufficiency and deficiency are common among continuous smokers with established mild-to-moderate chronic obstructive pulmonary disease, baseline 25(OH)D levels are not predictive of subsequent lung function decline.

KEYWORDS: Chronic obstructive pulmonary disease, smoking, spirometry, vitamin D

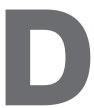
ata from the Third National Health and Nutrition Examination Survey (NHANES III) showed that in a cross-sectional sample of a general US population $(n=14,076)$, lower serum vitamin D levels were associated with lower forced expiratory volume in $1 \mathrm{~s}$ (FEV1) in a graded, "dose-dependent" fashion [1]. The results of this report from BLACK and SCRAGG [1] have spurred the hypothesis that low vitamin $\mathrm{D}$ levels may be a modifiable risk factor for impaired lung function and chronic obstructive pulmonary disease (COPD).

Vitamin D has long been recognised for its effects on calcium homeostasis and skeletal health. However, its nonskeletal effects have recently received increasing scientific attention, including hypotheses on its potentially beneficial effects in patients with COPD [2]. The mechanisms by which vitamin D levels might affect lung function are unclear. Potential explanations include effects on respiratory infection risk (via both innate and adaptive mechanisms) and lung tissue remodelling (via matrix metalloproteinases and other pathways) [2-5].
We sought to build upon the cross-sectional data of BLACK and SCRAGG [1] by using longitudinal data to further investigate vitamin $\mathrm{D}$ insufficiency as a risk factor for rapid lung function decline and COPD. We hypothesised that, among persons with mild COPD, those with rapid declines in longitudinal lung function would have lower baseline vitamin D levels compared to persons with minimal declines in longitudinal lung function. We tested this hypothesis with a nested, matched case-control study in the Lung Health Study (LHS) 3 cohort.

\section{MATERIALS AND METHODS}

\section{Study subjects}

Participants in this nested, matched case-control study were selected from LHS 3, an observational follow-up study of participants in the LHS trial, a 5 -yr, 10-centre, randomised trial of a smoking intervention and bronchodilators [6, 7]. Following the trial, study interventions were stopped, but most participants provided informed consent to participate in LHS 3 and agreed to return to study centres for a single long-term (LT) follow-up visit.
AFFILIATIONS

*Division of Pulmonary, Allergy,

Critical Care and Sleep Medicine,

${ }^{+}$Division of Biostatistics, University of Minnesota,

\#Pulmonary Section, Minneapolis Veterans Affairs Medical Center,

Minneapolis, MN, and

'Dept of Laboratory Medicine and Pathology, Mayo Clinic, Rochester MN, USA.

\section{CORRESPONDENCE}

K.M. Kunisaki

Minneapolis Veterans Affairs Medical Center

Pulmonary

$111 \mathrm{~N}$

1 Veterans Drive

Minneapolis

MN 55417

USA

E-mail: kunis001@umn.edu

Received:

Sept 152009

Accepted after revision:

June 102010

First published online:

July 012010 
5,887 participants enrolled in the original LHS trial, 4,517 participants enrolled in LHS 3 and 4,194 completed spirometry at an average of 6 yrs after LHS 3 enrolment. Thus, the followup rate in LHS 3 was 93\%. Detailed methods and results of LHS 3 have been published previously [8,9].

\section{Study design}

We conducted a nested, matched case-control study within the LHS 3 cohort. Stored blood was available only at the end of the original trial, at the year 5 (Y5) visit; blood was not stored from other visits in the original trial or at the LT follow-up visit. This Y5 specimen served as our baseline vitamin D assessment. Spirometry was available at the time of phlebotomy (at Y5) and at the LT visit. LHS 3 did not have any intermediate visits between the Y5 and LT visits (see online supplementary material for figure of design). We restricted our analysis to the 1,054 LHS 3 participants who were biochemically validated continuous smokers throughout all visits in the original LHS trial and still smoking at the LT visit. Cases were continuous smokers with the most rapid declines in FEV1 between the Y5 visit and the LT visit (rapid decliners). Controls were continuous smokers with the least decline in FEV1 in the same time period (slow decliners).

The primary human source of vitamin D is ultraviolet sunlight exposure, which will vary by season and by latitude (LHS study centres varied from as far south as Birmingham, AL, USA (latitude $33^{\circ} \mathrm{N}$ ) to as far north as Winnepeg, MB, Canada (latitude $49^{\circ} \mathrm{N}$ )). To control for these seasonal and latitude effects on vitamin D levels, we matched cases and controls on date of Y5 phlebotomy (to within 60 days) and clinical centre. We reasoned that if vitamin $\mathrm{D}$ affected rates of FEV1 decline, then differences in vitamin $\mathrm{D}$ levels should be greatest between persons with the greatest differences in rates of lung function decline. Therefore, we constructed an LHS 3 database query, such that the matched case-control pair with the largest difference in rates of FEV1 decline (as \% predicted) was selected as the first pair. This process was repeated sequentially and subsequent pairs had progressively smaller differences in rate of FEV1 decline between the cases and controls, while still remaining matched on date and clinical centre (see online supplementary material for figure of selection process). This selection process was continued until the desired sample size was reached.

\section{Methods}

Plasma was collected at the Y5 visit in a standardised fashion, and shipped on dry ice to the LHS 3 data coordinating center, where samples have been continuously stored at $-70^{\circ} \mathrm{C}$. Once cases and controls were identified for this particular study, plasma samples were thawed and plasma 25-hydroxyvitamin D $(25(\mathrm{OH}) \mathrm{D})$ assays were performed using liquid chromatographytandem mass spectrometry (LC-MS/MS; ThermoFisher Scientific, Franklin, MA, USA and Applied Biosystems/MDS Sciex, Foster City, CA, USA) in the laboratory of R.J. Singh at the Mayo Clinic (Rochester, MN, USA). Details of the 25(OH)D assays, including coefficients of variation, are provided in the online supplementary material.

Spirometry was performed in both the main trial and LT visit using the same rolling-seal spirometers (Spirotech 500; Spirotech, Atlanta, GA, USA) and the same spirometry quality control programme [10]. Measurements of FEV1 and forced vital capacity (FVC) were made before and $\geqslant 20 \mathrm{~min}$ after two puffs $(200 \mu \mathrm{g})$ of inhaled albuterol administered through a metered-dose inhaler. Our analysis was restricted to postbronchodilator measures. The largest single FEV1 and FVC were reported and converted to percentages of the predicted normal using the formulas of CRAPO et al. [11].

\section{Analysis}

The primary outcome was the paired difference in the baseline exposure variable (Y5 plasma 25(OH)D level) between matched rapid and slow decliners. Annual rates of lung function decline were calculated by subtracting spirometry values at the LT visit from the values at the $\mathrm{Y} 5$ visit and dividing by the time elapsed between the two measures.

Our sample size of 196 participants (98 pairs) provided 90\% power (two-tailed $\alpha=0.05$ ) to detect a difference of $2.4 \mathrm{ng} \cdot \mathrm{mL}^{-1}$ in baseline $25(\mathrm{OH}) \mathrm{D}$ levels between rapid and slow decliners using paired t-testing. We therefore had excellent power to detect small differences in $25(\mathrm{OH}) \mathrm{D}$ levels. Details of the sample size determination are provided in the online supplementary material.

As secondary analyses, we also investigated seasonal variation in $25(\mathrm{OH}) \mathrm{D}$ levels. Seasons were defined as winter (JanuaryMarch), spring (April-June), summer (July-September) and autumn (October-December). The seasonal 25(OH)D data were analysed using one-way ANOVA, corrected for multiple comparisons with a Bonferroni-adjusted $\mathrm{p}$-value significance level of 0.05 . We also conducted a conditional (paired) logistic regression analysis in which the outcome was rapid versus slow decline in FEV1 and which included the following covariates from the $\mathrm{Y} 5$ visit (in addition to total vitamin $\mathrm{D}$ level): age, sex, number of cigarettes smoked per day, FEV1 \% pred, FVC \% pred, bronchodilator response and methacholine response. We also included time from the Y5 visit to the LT visit.

All statistical analyses were performed using SAS 9.1 (SAS Institute, Cary, NC, USA) and STATA 9.2 (StatCorp, College Station, TX, USA). Figures were created using SigmaPlot 11.0 (Systat Software Inc., San Jose, CA, USA).

\section{RESULTS}

Rapid and slow decliners (cases and controls) were similar in Y5 age, sex distribution and smoking intensity (table 1). Most participants were Caucasian, due to the sample recruited in the original LHS trial, and there was a statistically significant difference in ethnicity of rapid and slow decliners. Matching resulted in a mean \pm SD difference in clinic visit days between rapid and slow decliners of $25 \pm 16.5$ days (range 0-60 days). The distribution of participants matched by clinical centre was as follows: Baltimore, MD, USA $(n=24)$, Birmingham, AL, USA $(n=10)$, Cleveland, OH, USA $(n=14)$, Detroit, MI, USA $(n=24)$, Los Angeles, CA, USA $(n=20)$, Pittsburgh, PA, USA $(n=24)$, Portland, OR, USA $(n=22)$, Rochester, MN, USA $(n=12)$, Salt Lake City, UT, USA $(n=18)$, and Winnipeg, MB, Canada $(n=28)$.

Our selection criteria for cases and controls resulted in clinically and statistically significant differences in the rate of FEV1 decline between rapid and slow decliners. While FEV1 was similar between rapid and slow decliners at the $\mathrm{Y} 5$ visit, rapid decliners had a mean FEV1 that was $>1 \mathrm{~L}$ worse than 


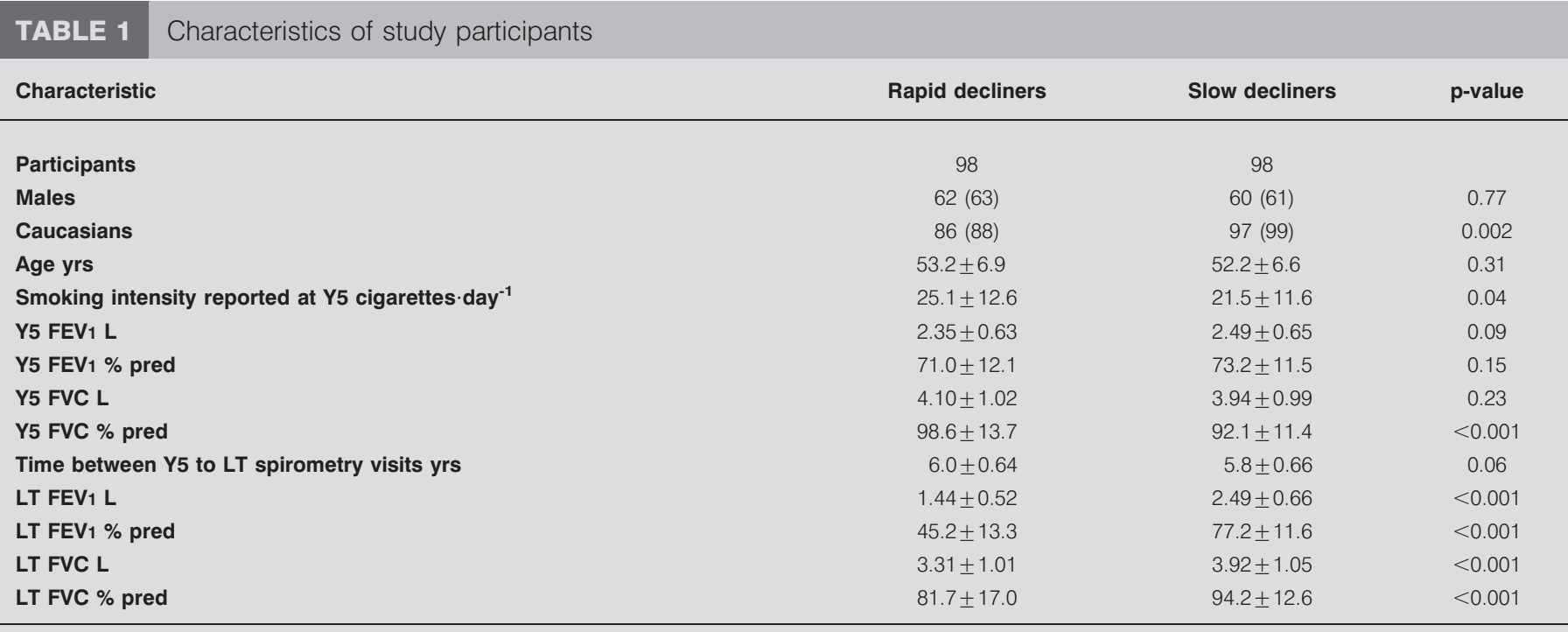

Data are presented as $n, n(\%)$ or mean $\pm S D$, unless otherwise stated. All participants were continuous smokers from the first visit in the main Lung Health Study trial to the long-term follow-up visit (LT). Y5: year 5 visit; FEV1: forced expiratory volume in $1 \mathrm{~s}$; \% pred: \% predicted; FVC: forced vital capacity.

slow decliners at the LT visit (table 1). This resulted in a rate of FEV1 decline of $-152 \mathrm{~mL} \cdot \mathrm{yr}^{-1}\left(-4.3 \% \mathrm{pred} \cdot \mathrm{yr}^{-1}\right)$ in $\mathrm{rapid}$ decliners versus $-0.3 \mathrm{~mL} \cdot \mathrm{yr}^{-1}\left(+0.7 \%\right.$ pred $\left.\cdot \mathrm{yr}^{-1}\right)$ in slow decliners $(\mathrm{p}<0.001)$ (table 2).

Despite the large differences in rate of FEV1 decline, and appropriate control of latitude (through matching on clinical centre) and time of year (through matching on date of phlebotomy), the difference in $\mathrm{Y} 525(\mathrm{OH}) \mathrm{D}$ level between rapid and slow decliners was not statistically significant $\left(25.0 \mathrm{ng} \cdot \mathrm{mL}^{-1}\right.$ versus $25.9 \mathrm{ng} \cdot \mathrm{mL}^{-1}$, respectively; $\mathrm{p}=0.54$ ) (table 2 ). Additional multiple regression and paired logistic regression analysis for baseline Y5 covariates also demonstrated no association between Y5 25(OH)D level and rapid or slow decliner status (see online supplementary material for details).

We applied current widely accepted definitions of vitamin D insufficiency and deficiency [12], which classify patients with $25(\mathrm{OH}) \mathrm{D}<20 \mathrm{ng} \cdot \mathrm{mL}^{-1}$ as vitamin D-deficient, and $\geqslant 20 \mathrm{ng} \cdot \mathrm{mL}^{-1}$ but $<30 \mathrm{ng} \cdot \mathrm{mL}^{-1}$ as vitamin D-insufficient. Applying such criteria, we found $35 \%(n=69)$ of this LHS 3 sample was vitamin D-insufficient and 31\% $(n=60)$ was vitamin D-deficient. Only $34 \% \quad(n=67)$ of the sample would currently be classified as vitamin D-sufficient, with levels $\geqslant 30 \mathrm{ng} \cdot \mathrm{mL}^{-1}$. $14(7 \%)$ participants had severe vitamin $\mathrm{D}$ deficiency, such that their $25(\mathrm{OH}) \mathrm{D}$ levels were $\leqslant 10 \mathrm{ng} \cdot \mathrm{mL}^{-1}$.

There was also significant seasonal variation in $25(\mathrm{OH}) \mathrm{D}$ levels (fig. 1 and online supplementary figure). As expected, $25(\mathrm{OH}) \mathrm{D}$ levels peaked in late summer, with a nadir observed in the winter months. The magnitude of the seasonal variation was both clinically and statistically significant, with a mean winter $25(\mathrm{OH}) \mathrm{D}$ level of $18.3 \mathrm{ng} \cdot \mathrm{mL}^{-1}$ compared to $31.7 \mathrm{ng} \cdot \mathrm{mL}^{-1}$ in the summer (Bonferroni-corrected $\mathrm{p}<0.001$ ). Of 48 samples drawn in the winter months, 46 (96\%) were under the recommended goal level of $\geqslant 30 \mathrm{ng} \cdot \mathrm{mL}^{-1}$.

\section{DISCUSSION}

We found no differences in baseline $25(\mathrm{OH}) \mathrm{D}$ levels between continuously smoking LHS 3 participants with rapid and slow declines in lung function over $\sim 6$ yrs of prospective follow-up. Therefore, our data do not support the notion that low $25(\mathrm{OH}) \mathrm{D}$ levels lead to faster rates of lung function decline.

Our study was primarily prompted by the study of BLACK and SCRAGG [1], which examined cross-sectional data from 14,076 NHANES III participants. They demonstrated a graded relationship between lower $25(\mathrm{OH}) \mathrm{D}$ levels and lower lung

TABLE 2 Comparison of lung function decline and vitamin D status between rapid decliners and slow decliners, matched on date of phlebotomy (to within 60 days) and clinical centre

\begin{tabular}{|c|c|c|c|}
\hline Characteristic & Rapid decliners & Slow decliners & p-value \\
\hline Participants & 98 & 98 & \\
\hline Rate of FEV1 decline from Y5 to LT \% pred.yr ${ }^{-1}$ & $-4.3 \pm 1.2$ & $+0.7 \pm 0.7$ & $<0.0001$ \\
\hline Y5 25(OH)D levels $\mathrm{ng} \cdot \mathrm{mL}^{-1}$ & $25.0 \pm 10.4$ & $25.9 \pm 10.2$ & 0.54 \\
\hline
\end{tabular}

Data are presented as $n$ or mean \pm SD, unless otherwise stated. $p$-values calculated using paired t-testing. FEV1: forced expiratory volume in $1 \mathrm{~s}$; Y5: year 5 visit; $L T$ : longterm follow-up visit; \% pred: \% predicted; 25(OH)D: 25-hydroxyvitamin D. 


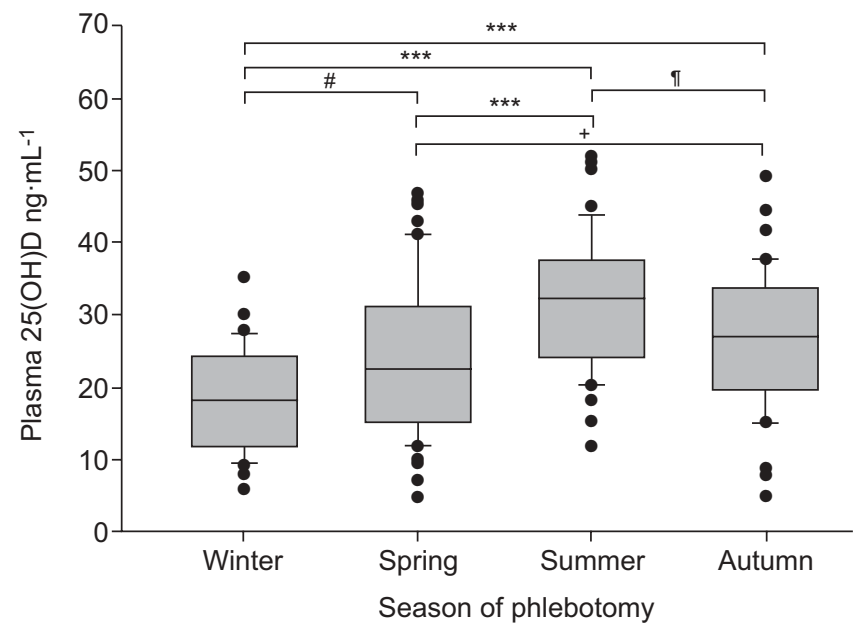

FIGURE 1. Box plots of 25-hydroxyvitamin D $(25(\mathrm{OH}) \mathrm{D})$ levels by season (winter: January-March; spring: April-June; summer: July-September; autumn: October-December). - : median; 1 : interquartile range; whiskers: range; outliers. Statistical testing of means listed in table 3 (one-way ANOVA with Bonferroni-corrected $p$-value) are shown. ${ }^{* \star *}: p<0.001 ;{ }^{\#}: p=0.11 ;{ }^{~}$ : $p=0.055$; $+: p=1.000$.

function, such that those in the lowest 25(OH)D quintile $\left(\leqslant 16.2 \mathrm{ng} \cdot \mathrm{mL}^{-1}\right)$ had a mean FEV1 that was $126 \mathrm{~mL}$ lower than those in the highest quintile $\left(\geqslant 34.3 \mathrm{ng} \cdot \mathrm{mL}^{-1}\right)$, after adjusting for sex, age, ethnicity, body mass index and cigarette smoking. Among a small subgroup with self-reported emphysema $(n=251)$, the differences were even greater, such that when comparing those with $25(\mathrm{OH}) \mathrm{D} \leqslant 16.2 \mathrm{ng} \cdot \mathrm{mL}^{-1}$ to those with $\geqslant 34.3 \mathrm{ng} \cdot \mathrm{mL}^{-1}, \mathrm{FEV} 1$ was $344 \mathrm{~mL}$ worse in the low $25(\mathrm{OH}) \mathrm{D}$ group. The actual spirometry values from these 251 patients were not reported, so confirmation of COPD and assessment of COPD severity could not be made. While intriguing, a major limitation of these data is the cross-sectional nature of NHANES data. To our knowledge, ours is the first study examining relationships between baseline $25(\mathrm{OH}) \mathrm{D}$ levels and subsequent prospective, longitudinal rates of lung function decline.

Our study design allowed us to compare two groups of COPD patients of significant clinical interest: those who continuously smoke and have rapid lung function decline (rapid decliners) and those who continuously smoke, yet have preserved lung function over time (slow decliners). Because smoking is controlled for in both of these groups, we were able to investigate the hypothesis that the rapid decliners would have lower $25(\mathrm{OH}) \mathrm{D}$ levels as one potential mechanism by which their lung function rapidly declines. However, our data do not support this hypothesis.

Our study has several strengths. The longitudinal assessment of lung function was rigorously standardised, with the same equipment and procedures used by experienced study staff (who had performed annual spirometry for 5 yrs prior to the Y5 measure in this study). Our matching criteria and the seasonal variation observed suggest that misclassification of $25(\mathrm{OH}) \mathrm{D}$ levels is unlikely. We had excellent power to detect small differences in $25(\mathrm{OH}) \mathrm{D}$ levels: $90 \%$ power to detect a difference as small as $2.4 \mathrm{ng} \cdot \mathrm{mL}^{-1}$. It seems unlikely that a difference any smaller than this could explain differences in rates of subsequent lung function decline.

Our study has several important limitations. One limitation is that assessment of $25(\mathrm{OH}) \mathrm{D}$ levels was only possible from a single study visit. Therefore, this single assessment may not be fully reflective of an individual's overall vitamin D status. For example, a wintertime assessment could be a poor indicator of overall vitamin D status throughout the year, especially in more extreme latitudes. We attempted to correct for seasonal variation and latitude effects as best as we could by matching rapid and slow decliners on date of phlebotomy and clinical centre, but this cannot correct for seasonal changes, which might vary significantly both within and between individuals. We were also unable to assess whether or not the presence of low 25(OH)D levels at $\mathrm{Y} 5$ were associated with persistent low levels at the LT follow-up visit, as there was no blood draw at the LT follow-up visit. It is possible that some participants might have begun activities during that time interval which could have affected their subsequent $25(\mathrm{OH}) \mathrm{D}$ levels. For example, participants could have begun vitamin D supplementation and subsequently increased their 25(OH)D levels after the Y5 visit. Conversely, they could have begun using sunscreen products, which could have decreased their 25(OH)D levels after the Y5 visit.

Another limitation of our data is that these analyses were restricted to continuous smokers with evidence of mild-tomoderate COPD at baseline. Because smoking has such a significant impact on rate of lung function decline, smoking is important to control for in a study such as ours. We chose to restrict our analysis to continuous smokers in order to focus on those COPD patients at greatest risk of progressive lung

TABLE 3 Year 5 plasma 25-hydroxyvitamin D (25(OH)D) levels by season

\begin{tabular}{lcccc} 
Characteristic & Winter & Spring & Summer & Autumn \\
\hline Participants & 48 & 51 & 57 & 40 \\
25(OH)D $\mathbf{n g} \cdot \mathbf{m L}^{-1}$ & $18.3 \pm 7.0$ & $24.1 \pm 10.3$ & $31.7 \pm 9.2$ & $26.8 \pm 9.6$ \\
Normal vitamin D level $^{\#}$ & $2(4)$ & $16(31)$ & $34(60)$ & $15(38)$ \\
Vitamin D-insufficient $^{*}$ & $19(40)$ & $15(29)$ & $20(35)$ & $3(5)$ \\
Vitamin D-deficient $^{+}$ & $27(56)$ & $20(39)$ & $3(25)$ & $10(25)$ \\
\hline
\end{tabular}

Data are presented as $\mathrm{n}$, mean \pm SD or $\mathrm{n}(\%)$. Seasons were defined as winter (January-March), spring (April-June), summer (July-September) and autumn (OctoberDecember). See figure 1 for $\mathrm{p}$-values from pairwise statistical testing of mean $25(\mathrm{OH}) \mathrm{D}$ levels by season. ${ }^{\#}: \geqslant 30 \mathrm{ng} \cdot \mathrm{mL}^{-1} ;{ }^{\bullet}: \geqslant 20$ but $<30 \mathrm{ng} \cdot \mathrm{mL}^{-1} ;{ }^{+}:<20 \mathrm{ng} \cdot \mathrm{mL}^{-1}$ 
function decline and reduce effects of variables other than vitamin $\mathrm{D}$ (e.g. intermittent smoking) that might also affect rate of lung function decline. Thus, we can not extrapolate these findings to nonsmokers or to intermittent smokers. We also can not extrapolate these findings to persons without COPD confirmed by spirometry, nor to persons with very advanced COPD.

We feel it important to highlight the high prevalence of vitamin D insufficiency and deficiency we found, such that only $34 \%$ of these LHS 3 participants had $25(\mathrm{OH}) \mathrm{D}$ levels that would currently be considered as adequate. In winter, we found only two out of $4825(\mathrm{OH}) \mathrm{D}$ measurements to be in the accepted normal range. RIANCHO et al. [13] studied 44 males with COPD (mean FEV1 39\% pred) between 1983 and 1985, and showed the mean $25(\mathrm{OH}) \mathrm{D}$ level was $<10 \mathrm{ng} \cdot \mathrm{mL}^{-1}$ for most of the year, with peak mean $25(\mathrm{OH}) \mathrm{D}$ level in late summer still $<20 \mathrm{ng} \cdot \mathrm{mL}^{-1}$. They measured $25(\mathrm{OH}) \mathrm{D}$ using a competitive protein-binding assay after high-performance liquid chromatography purification, a method that is now rarely used, so a direct comparison to more current $25(\mathrm{OH}) \mathrm{D}$ assay methods may be limited. SHANE et al. [14] reported a mean $25(\mathrm{OH}) \mathrm{D}$ level of $20 \mathrm{ng} \cdot \mathrm{mL}^{-1}$ in 28 patients with COPD awaiting lung transplantation between 1993 and 1995. 10 (36\%) of these patients had levels $\leqslant 10 \mathrm{ng} \cdot \mathrm{mL}^{-1}$. FORLI et al. [15] reported vitamin D deficiency $\left(<20 \mathrm{ng} \cdot \mathrm{mL}^{-1}\right)$ in $>50 \%$ of 71 consecutive nonsmoking patients (of whom 46 had COPD) undergoing lung transplantation evaluation between 1993 and 1998.

These data are of particular concern in light of recent NHANES data demonstrating that, between the surveys conducted in 1988-1994 and 2001-2004, the mean population 25(OH)D level decreased by $6 \mathrm{ng} \cdot \mathrm{mL}^{-1}$ and the percentage with inadequate $25(\mathrm{OH}) \mathrm{D}$ levels $\left(<30 \mathrm{ng} \cdot \mathrm{mL}^{-1}\right)$ increased from 55 to $77 \%$ [16]. Because our 25(OH)D data are based on samples collected between 1991 and 1994, it seems likely that the current prevalence of inadequate $25(\mathrm{OH}) \mathrm{D}$ levels in patients with mild-to-moderate COPD is even higher than the $66 \%$ we found.

In support of this, FRANCO et al. [17] recently reported a spring 2005 mean $25(\mathrm{OH}) \mathrm{D}$ level of $20.8 \mathrm{ng} \cdot \mathrm{mL}^{-1}$ in a small cohort of 49 Brazilian patients with mostly mild and moderate COPD. Of these 49 patients, only three $(6 \%)$ had $25(\mathrm{OH}) \mathrm{D}$ levels $\geqslant 30 \mathrm{ng} \cdot \mathrm{mL}^{-1}, 29(59 \%)$ were vitamin D-insufficient and 17 (35\%) were vitamin D-deficient. JANSSENS et al. [18] also recently reported that, among 262 Belgian patients with COPD, the mean $25(\mathrm{OH}) \mathrm{D}$ level was $19.9 \mathrm{ng} \cdot \mathrm{mL}^{-1}$ and $52 \%$ were vitamin D-deficient, with levels $<20 \mathrm{ng} \cdot \mathrm{mL}^{-1}$.

Our cohort also demonstrated significant seasonal variation in $25(\mathrm{OH}) \mathrm{D}$ levels, which varied around the accepted cut-off points for normal, insufficient and deficient levels. As such, there was a substantial seasonal shift in the distribution of participants classified as normal or vitamin D-deficient. While these blood samples from 1991-1994 are no longer a contemporary assessment, clinicians and researchers may need to consider the substantial effect of seasonality on $25(\mathrm{OH}) \mathrm{D}$ measures. It is important to note that LHS 3 participants were generally quite healthy, with mostly mild COPD. One might hypothesise that in patients with more severe COPD, there may be less of a seasonal effect, due to their being more confined to the home and, hence, less exposed to sunlight. However, we are unaware of any contemporary data to either support or refute such a hypothesis. In addition, the mechanisms leading to vitamin D insufficiency/deficiency may be quite complex. Dietary vitamin D intake in patients with COPD has been shown to be low [19], but multiple other mechanisms may lead to inadequate vitamin D status [12].

Although we found no association between 25(OH)D levels and subsequent rates of lung function decline, patients with COPD suffer from many comorbidities potentially associated with low 25(OH)D levels. The one COPD comorbidity with well-studied links to low 25(OH)D levels is osteoporosis [20]. Multiple other COPD complications and comorbidities have been linked to vitamin $\mathrm{D}$ insufficiency, including respiratory infections [21-23], cardiovascular disease [24, 25] and muscle dysfunction [26, 27]. However, it is important to note that there are no clinical trial data to support to the hypothesis that improving 25(OH)D levels in patients with COPD will improve any of these COPD comorbidities, but these remain topics requiring further investigation.

In conclusion, although we found a high prevalence of low $25(\mathrm{OH}) \mathrm{D}$ levels in continuous smokers with established mild and moderate COPD, we found no difference between baseline $25(\mathrm{OH}) \mathrm{D}$ levels among those with subsequent rapid declines in lung function and slow declines in lung function. Our data suggest that normalisation of $25(\mathrm{OH}) \mathrm{D}$ levels is not likely to affect subsequent rates of lung function decline in such patients.

\section{SUPPORT STATEMENT}

The study was supported by the Minnesota Medical Foundation (faculty research award \#3857-9227-08 to K.M. Kunisaki) and National Institutes of Health (K12 RR023247 to K.M. Kunisaki and UL1 RR024150 to the Mayo Clinic).

\section{STATEMENT OF INTEREST}

None declared.

\section{ACKNOWLEDGEMENTS}

We thank H. Blair (Mayo Clinic, Rochester, MN, USA) for technical assistance with the vitamin D assays, and H. Voelker and A. Boggess (both University of Minnesota, Minneapolis, MN, USA) for assistance with the identification and retrieval of samples. We also thank the Upper Midwest CTSA Consortium for fostering this study's institutional collaboration.

\section{REFERENCES}

1 Black PN, Scragg R. Relationship between serum 25-hydroxyvitamin D and pulmonary function in the Third National Health and Nutrition Examination Survey. Chest 2005; 128: 3792-3798.

2 Janssens W, Lehouck A, Carremans C, et al. Vitamin D beyond bones in chronic obstructive pulmonary disease: time to act. Am J Respir Crit Care Med 2009; 179: 630-636.

3 Koli K, Keski-Oja J. 1 $\alpha$,25-dihydroxyvitamin D3 and its analogues down-regulate cell invasion-associated proteases in cultured malignant cells. Cell Growth Differ 2000; 11: 221-229.

4 Bao BY, Yeh SD, Lee YF. 1 $\alpha, 25$-dihydroxyvitamin D3 inhibits prostate cancer cell invasion via modulation of selective proteases. Carcinogenesis 2006; 27: 32-42.

5 Timms PM, Mannan N, Hitman GA, et al. Circulating MMP9, vitamin D and variation in the TIMP-1 response with VDR genotype: mechanisms for inflammatory damage in chronic disorders? QJM 2002; 95: 787-796. 
6 Connett JE, Kusek JW, Bailey WC, et al. Design of the Lung Health Study: a randomized clinical trial of early intervention for chronic obstructive pulmonary disease. Control Clin Trials 1993; 14: 3S-19S.

7 Anthonisen NR, Connett JE, Kiley JP, et al. Effects of smoking intervention and the use of an inhaled anticholinergic bronchodilator on the rate of decline of FEV1. The Lung Health Study. JAMA 1994; 272: 1497-1505.

8 Anthonisen NR, Connett JE, Murray RP. Smoking and lung function of lung health study participants after 11 years. Am J Respir Crit Care Med 2002; 166: 675-679.

9 Anthonisen NR, Skeans MA, Wise RA, et al. The effects of a smoking cessation intervention on 14.5-year mortality: a randomized clinical trial. Ann Intern Med 2005; 142: 233-239.

10 Enright PL, Johnson LR, Connett JE, et al. Spirometry in the Lung Health Study. 1. Methods and quality control. Am Rev Respir Dis 1991; 143: 1215-1223.

11 Crapo RO, Morris AH, Gardner RM. Reference spirometric values using techniques and equipment that meet ATS recommendations. Am Rev Respir Dis 1981; 123: 659-664.

12 Holick MF. Vitamin D Deficiency. N Engl J Med 2007; 357: 266-281.

13 Riancho JA, Gonzalez Macias J, Del Arco C, et al. Vertebral compression fractures and mineral metabolism in chronic obstructive lung disease. Thorax 1987; 42: 962-966.

14 Shane E, Silverberg SJ, Donovan D, et al. Osteoporosis in lung transplantation candidates with end-stage pulmonary disease. Am J Med 1996; 101: 262-269.

15 Forli L, Halse J, Haug E, et al. Vitamin D deficiency, bone mineral density and weight in patients with advanced pulmonary disease. J Intern Med 2004; 256: 56-62.

16 Ginde AA, Liu MC, Camargo CA Jr. Demographic differences and trends of vitamin D Insufficiency in the US Population, 1988-2004. Arch Intern Med 2009; 169: 626-632.
17 Franco CB, Paz-Filho G, Gomes PE, et al. Chronic obstructive pulmonary disease is associated with osteoporosis and low levels of vitamin D. Osteoporos Int 2009; 20: 1881-1887.

18 Janssens W, Bouillon R, Claes B, et al. Vitamin D deficiency is highly prevalent in COPD and correlates with variants in the vitamin D-binding gene. Thorax 2010; 65: 215-220.

19 de Batlle J, Romieu I, Antó JM, et al. Dietary habits of firstly admitted Spanish COPD patients. Respir Med 2009; 103: 1904-1910.

20 Cranney A, Weiler HA, O'Donnell S, et al. Summary of evidencebased review on vitamin D efficacy and safety in relation to bone health. Am J Clin Nutr 2008; 88: 513S-519S.

21 Ginde AA, Mansbach JM, Camargo CA Jr. Vitamin D, respiratory infections, and asthma. Curr Allergy Asthma Rep 2009; 9: 81-87.

22 Laaksi I, Ruohola J, Tuohimaa $\mathrm{P}$, et al. An association of serum vitamin D concentrations $<40 \mathrm{nmol} \cdot \mathrm{L}^{-1}$ with acute respiratory tract infection in young Finnish men. Am J Clin Nutr 2007; 86: 714-717.

23 Aloia JF, Li-Ng M. Re: epidemic influenza and vitamin D. Epidemiol Infect 2007; 135: 1095-1096.

24 Kendrick J, Targher G, Smits G, et al. 25-Hydroxyvitamin D deficiency is independently associated with cardiovascular disease in the Third National Health and Nutrition Examination Survey. Atherosclerosis 2009; 205: 255-260.

25 Lee JH, O'Keefe JH, Bell D, et al. Vitamin D deficiency: an important, common, and easily treatable cardiovascular risk factor? J Am Coll Cardiol 2008; 52: 1949-1956.

26 Bischoff-Ferrari HA, Dietrich T, Orav EJ, et al. Higher 25hydroxyvitamin D concentrations are associated with better lower-extremity function in both active and inactive persons aged $\geqslant 60$ y. Am J Clin Nutr 2004; 80: 752-758.

27 Sato Y, Iwamoto J, Kanoko T, et al. Low-dose vitamin D prevents muscular atrophy and reduces falls and hip fractures in women after stroke: a randomized controlled trial. Cerebrovasc Dis 2005; 20 : 187-192. 